\title{
LA BREBIS PRODUCTRICE DE LAIT ET FACTEUR ÉCONOMIQUE
}

par

\author{
Dr VIEIRA DE SA
}

Médecin vétérinaire. Ancien boursier du Gouvernement portugais à l'Université de Reading (Angleterre)

Un des problèmes les plus importants qui préoccupe tous les peuples du monde, et plus particulièrement ceux de l'Europe est indubitablement, celui de la production du lait, non seulement en ce qui concerne la consommation en nature, mais aussi en ce qui a rapport à la fabrication de laitages.

Si, en effet, c'est sur la boviniculture qu'il faut poser les prémices fondamentales pour la résolution de ce si important problème, nous ne pouvons ni ne devons oublier, toutefois la précieuse contribution qui nous a été offerte par d'autres espèces, parmi lesquelles se trouve en premier plan, l'espèce ovine.

Il faut se rappeler que, jusqu'à présent il n'a pas été possible, même en employant une substitution quelconque, de se passer de la laine produite par ces animaux, ce qui nous porte à les considérer - et chaque fois davantage - comme indispensables à la vie et à l'économie des sociétés humaines. En face de cette réalité, il faut accepter implicitement que, si l'existence de la brebis est une nécessité, dont nous ne pouvons nous passer, il faut en profiter au maximum de ses possibilités.

En nous plaçant sous ce point de vue, et considérant l'aspect de la production laitière, on vérifie que la règle de cette utilisation intégrale si désirable n'a pas été toujours suivie.

Comme on le sait, l'exploration ovine peut être faite en extension et en intensité. Au premier cas se rattache plus ou moins l'exclusivité de la production lainière (1) tandis qu'au second se rattache la production mixte "laine-lait" cette dernière fonction prédominant parfois. Entre ces deux points extrêmes se trouvent tous les autres aspects où les deux productions se rejoignent ou se séparent plus ou moins.

Comment expliquer cette diversité d'aspeets d'exploitation de la brebis ?

Parlons d'abord de l'incompatibilité de fonctions. Cette incom patibilité entre la production de lait et la production de laine

(1) Il est évident que le fait de ne pas se référer à la production de la viande ne témoigne en aucune façon d'un manque d'intérêt. Cette production coexiste dans toutes les circonstances, quoiqu'elle puisse dans certains cas, prendre un caractère tout à fait spécialisé. 
existe-t-elle lorsqu'on considère la question au point de vue physiologique?

Si l'on met de côté quelques conceptions génétiques qui pourraient exister sur l'aptitude fonctionnelle des races, ce que l'on observe dans les exemples connus, démontre précisément l'inexistence de cette incompatibilité.

Ainsi, au Portugal, les meilleures brebis productrices de laine, en qualité et en quantité, sont parfois, simultanément, les meilleures productrices de lait.

Prenons des exemples :

Des brebis " bordaleiras communs » à laine fine (croisée fine I-II) fournissent les meilleures productrices de lait connues dans cette espèce. En effet, des contrôles laitiers effectués sur plus de 2.000 têtes ont donné une moyenne de production approximative de 120 kilogrammes, pour 220 jours environ, le record individuel étant de 297 kilogrammes en 250 jours, soit une moyenne de $1 \mathrm{~kg} .190$ par jour.

Les meilleurs types de laine mérinos portugaise (mérinos 105110) sont fournis par un groupe de brebis d'Idanha dont la production de lait n'est pas estimée à moins de 40 kilogrammes en 110 jours.

Et même dans la "race mérinos » dont la zone de dispersion, au Portugal, est au Sud, les productions de lait ne descendent généralement pas au-dessous de 20 kilogrammes en 110 jours et on trouve des troupeaux qui, continuant à maintenir leur bon type de "laine mérinos ", grâce à une judicieuse sélection, atteignent des productions de lait de 40 kilogrammes ou même davantage. Ceci nous apparaît d'autant plus exceptionnel que nous nous rappelons que la "race mérinos " est considérée comme une très mauvaise race laitière, de telle sorte que, dans l'Espagne voisine, on n'a pas même l'habitude de la traire.

Disons plutôt - et c'est ainsi que la question doit être posée que la production de laine et la production de lait étant deux fonetions extrêmement exigeantes en azote, l'animal souffrira dans son équilibre physiologique, lorsqu'il n'aura pas une alimentation copieuse pouvant garantir une bonne compensation protéique et ceci d'autant plus que nous l'exploitons davantage.

Dans ce cas, il y a, sans doute, incompatibilité, due surtout à des facteurs de milieu, et au facteur alimentaire en première place.

Nous ne devons pas, d'ailleurs, oublier l'importance que la gymnastique fonctionnelle représente par rapport à une plus grande productivité de la glande mammaire. Si cette gymnastique ne se fait pas ou si elle se fait mal, à quoi faudra-t-il s'attendre sinon à ce que sa production se chiffre très bas ou même à ce qu'elle disparaisse 
totalement, ce qui se produit pour la brebis mérinos depuis longtemps déjà-?

Toutes ces considérations nous portent done à conclure que l'on pourra, dans l'avenir, utiliser la brebis comme productrice de lait, mieux qu'on ne l'a fait jusqu'à présent. On contribuera ainsi à la résolution $\mathrm{du}$ problème alimentaixe du Monde, prineipalement en ce qui concerne les protéines de première classe.

Le lait de brebis est particulièrement utilisé pour la fabrication du fromage. Il faut tenir compte de l'énorme importance de la production de ce laitage, non seulement parce qu'il représente au point de vue alimentaire, mais aussi par son intérêt économique dans toutes les régions pastorales de l'Europe, plus particulièrement dans les pays du basssin de la Méditerranée, intérêt qui peut dépasser considérablement celui que l'industrialisation du lait de vache nous offre.

En effet, au Portugal, par exemple, la quantité de fromage de brebis produite annuellement est estimée à 6.000.000 de kilogrammes environ, six fois plus que la production du fromage de lait de vache. Cette quantité est donnée par 1.050 .000 têtes, soit $27 \%$ de l'effectif ovin total, ce qui se traduit par une moyenne de production par tête de 34 litres de lait par lactation.

En Espagne, la production de fromage de brebis ne doit pas être très loin des 14.500.000 kilogrammes, et que je sache, il n'y a pas là d'industrialisation de lait de vache qui vaille référence. Ceci correspond à l'existence de 3.500 .000 têtes de brebis en production laitière, soit une moyenne de 25 litres par tête, revenu bien inférieur à celui prévu pour le Portugal.

En Slovaquie la production d'avant guerre était de $\mathbf{3 . 0 0 0 . 0 0 0}$ kilogrammes annuellement.

Nous tournant vers d'autres latitudes nous mentionnerons l'Islande où, selon les informations que j'ai recueillies, le principal animal laitier est représenté précisément par la brebis ; on en compte près de 700.000 têtes tandis qu'il n'y a que 34.600 vaches.

Je n'ai pas de détails sur les travaux que les Hollandais sont en train de faire sur les "Frisies ", mais je sais que leurs études s'orientent précisément vers la production laitière, en recherchant probablement en même temps, le perfectionnement de la laine.

La contribution de la brebis dans la production mondiale du lait ainsi démontrée par ces quelques exemples qui me semblent concluants, on peut se demander si le sujet ne mériterait pas d'être étudié de plus près afín de rendre possible une augmentation de la production laitière par le développement de l'élevage des brebis. Les conditions alimentaires et économiques des peuples en seraient ainsi 
améliorées et ceci sans préjudice pour la laine, puisqu'il ne semble pas y avoir de danger si la nourriture des bêtes est suffisante.

Le fromage de brebis subit une très grande demande sur tous les marehés par son goût particulier, mais, sans compter ses qualités sapides, il a en outre une valeur nutritive généralement bien plus importante que celle du fromage de vache.

Il y a une grande variété de fromages de brebis et quelques-uns sont de renommée internationale. Parmi les meilleurs on compte le "Serra ", d'origine portugaise, dont la fabrication encore ménagère ne garantit pas une qualité constante.

Ces considérations, qui m'ont paru suffisamment intéressantes ont pour but de faire un appel en faveur de l'augmentation de la production de lait de brebis.

Dans ce sens il faudrait :

Appliquer avec plus de généralisation au bétail ovin le contrôle laitier depuis si longtemps en usage courant pour la sélection du bétail bovin laitier ;

Orienter plus judicieusement l'exploitation des troupeaux, ne méprisant point, comme on a fait jusqu'ici, parfois, le sens de la production de lait;

Essayer, autant que possible, d'harmoniser les deux fonctions "laine-lait ", dès que les ressources alimentaires ne rendent pas le problème insoluble;

Intensifier les études zootechniques aux postes scientifiques d'élevage, dans le but d'améliorer chaque fois plus la capacité productrice de cette espèce en laine et en lait ;

Divulguer les principes techniques d'application pratique servant ces objectifs ;

Intensifier la production des coopératives d'oviniculteurs comme base pour le contrôle laitier et dans le but de centraliser la fabrication des fromages, afin que ceux-ci puissent être industrialisés selon des procédés modernes.

Je suggère, finalement que, dans les futurs Congrès Internationaux de Laiterie, fonctionne une section où seront traités exclusivement des problèmes concernant la production et l'industrialisation du lait de brebis, ainsi que celles du lait de chèvre. 